\title{
Process model based development of disassembly tools
}

U Rebafka, G Seliger*, A Stenzel and B R Zuo

Institute for Machine Tools and Factory Management, Technical University of Berlin, Germany

\begin{abstract}
Disassembly processes require flexible tools for loosening and handling operations. Today, disassembly processes demand a great deal of manual labour and a vast variety of tools. Partly destructive tools which generate and use new acting surfaces are able to increase the economic viability owing to their flexibility and their promotion of the reuse of components. This article describes selected methods of acting surface generation and their application for prototypical tools.
\end{abstract}

Keywords: disassembly, disassembly tools, joint connection, handling, plastic housing, process modelling

\section{NOTATION}

$b_{\mathrm{s}}$

$b_{\mathrm{w}}$

$d$

$D$

$e_{r}, e_{\varphi}, e_{z}$

$\dot{E}$

$\dot{E}_{\mathrm{B}}$

$\dot{E}_{\mathrm{BI}}$

$\dot{E}_{\mathrm{BO}}$

$\dot{E}_{\mathrm{BS}}$

$\dot{E}_{\mathrm{H}}$

$\dot{E}_{\mathrm{HI}}$

$\dot{E}_{\mathrm{HO}}$ coefficient of screw pitch

width of wedge

minimum diameter of the screwnail

maximum diameter of the screwnail

unit vector of coordinate axes of

cylindrical coordinate system (CYCS)

total rate of energy dissipation

rate of energy dissipated owing to plastic

flow in the groove of the screwnail body

rate of energy dissipated owing to friction

on the inner cylindrical surface of the

screwnail body

rate of energy dissipated owing to the

velocity discontinuity on the outer

cylindrical surface of the screwnail body

rate of energy dissipated owing to friction

along the groove interface of the

screwnail body

rate of energy dissipated owing to

plastic flow in the groove of the screwnail head

rate of energy dissipated owing to friction on the inner cone surface of the screwnail head

rate of energy dissipated owing to the velocity discontinuity on the outer cone surface of the screwnail head
$\dot{E}_{\mathrm{HS}}$

$\dot{E}_{\mathrm{S}}$

$F_{\text {a }}$

$F_{\mathrm{s}}$

$h_{0}$

H

$k$

$m_{x}$

$p_{\mathrm{r}}$

$P$

$R$

$s_{\mathrm{n} r}$

$s_{\mathrm{r}}$

$s_{\text {tr }}$

$S, S_{V}, S_{T}$

$T_{\mathrm{i}}$

$u, u_{r}, u_{\varphi}, u_{z}$

$U$

$v, v_{r}, v_{\varphi}, v_{z}$

V

$w, w_{r}, w_{\varphi}, w_{z}$

$w_{\eta}$

$x, y, z$

$z_{\mathrm{d}}$

$\alpha_{\mathrm{r}}, \alpha_{1}$ rate of energy dissipated owing to friction along the groove interface of the screwnail head

rate of energy dissipated owing to the velocity discontinuity on the ring-like plane separating the screwnail head from the body axial indentation force side indentation force length of the screwnail indentation depth yield stress in shear energy coefficients with respect to $\dot{E}_{x}$ (where $x=\mathrm{H}, \mathrm{B}, \mathrm{S}, \mathrm{HO}, \mathrm{BO}, \mathrm{HI}, \mathrm{BI}, \mathrm{HS}$, BS) hydrostatic pressure in region ADE screw pitch screw radius normal component of $v_{r}$ speed of material flow on right side wedge-tip component of $U$ area of the external and internal surface indentation torque velocity of the screwnail indentation velocity velocity of plastic material volume of the plastic material relative velocity between plastic material and screw tangential relative velocity between screw and plastic material

The MS was received on 15 June 2000 and was accepted after revision for publication on 8 February 2001.

* Corresponding author: Institute for Machine Tools and Factory Management (IWF), Technical University of Berlin, Pascalstraße 8-9, D-10587 Berlin, Germany.
Cartesian coordinate system (CACS)

indentation depth

angle of right/left centred fan 


$\begin{array}{ll}\beta_{\mathrm{r}}, \beta_{1} & \text { right/left semi-wedge angle } \\ \delta_{\mathrm{r}}, \delta_{1} & \text { lip angle on right/left side } \\ \dot{\varepsilon}_{i j} & \text { strain rate tensor under CACS } \\ \eta & \text { inclined angle } \\ \lambda_{i} & \text { velocity coefficients } \\ \mu & \text { coefficient of friction between the wedge } \\ & \text { and indented block } \\ \sigma_{\mathrm{r}}, \sigma_{1} & \text { normal stress on right/left side } \\ \tau, \tau_{\mathrm{r}}, \tau_{1} & \text { frictional stress on right/left side } \\ \varphi_{\mathrm{r}}, \varphi_{1} & \text { angle of material flow on right/left side } \\ \omega & \text { rotational velocity }\end{array}$

\section{INTRODUCTION}

The worldwide increase in waste production on the one hand and the restriction of natural resources on the other hand make recycling of worn-out products a necessity. Disassembly of consumer and industrial goods offers advantages such as material recovery, reuse of components and separation of hazardous materials compared with other recycling technologies. A drawback for disassembly is that it still lacks economic efficiency. Today, disassembly is mainly done manually, rarely supported by adequate tools, which results in high labour costs.

Unfortunately, the present recycling has to deal with products usually not designed for disassembly. This hampers the creation of added value during the recycling process [1]. Highly flexible tools are required owing to the diverse joining techniques, limited information about product geometry and unpredicted alterations. Alterations can be wear, rust of components and joints and replacement of components [2]. Up to now, ordinary assembly and manufacturing tools not designed for disassembly purposes have been used. Disassembly requires numerous tools for different joints, the recognition of joints, selection of appropriate tools and frequent tool changes. Forming new acting surfaces by wedge indentation is a cost effective approach to overcoming geometric uncertainties in the disassembly process. This paper presents general methods for generating and using new acting surfaces.

\section{GENERAL APPROACH: ACTING SURFACE GENERATION}

Loosening of joints as well as handling and fixing are operations of major importance during the disassembly of used products. Up to now, forces and torques have been transmitted to these operations via existing acting surfaces such as slots in screw heads. Tools and devices have to be adapted to the wide variety of geometrical shapes of joining elements and components. In order to meet high flexibility demands for disassembly processes, tools should be applied to a wide range of geometries. New end-effectors have to be developed that create their own acting surface at the beginning of the loosening, handling or fixing process, thus being independent of a wide variety of shapes (Fig. 1).

The approach of acting surface generation has been implemented for prototypical tools for the loosening of screws and the handling of products and components. Firstly, the processes according to DIN 8583, master shaping, forming, separating, jointing and coating [3], have been analysed for their suitability. Considering constraints such as accessibility and processing time, the solution space has been narrowed to forming, separating and assembling work. In particular, processes such as indentation forming, drilling, milling, sawing and abrasive cutting, as well as welding, soldering and bonding, have been taken into account. Within this group, indentation forming [4] according to DIN 8580 has been favoured as the most appropriate process.

From this subgroup, sudden indentation was chosen for the loosening of screws. The introduced impulse energy will be transformed into plastic deformation

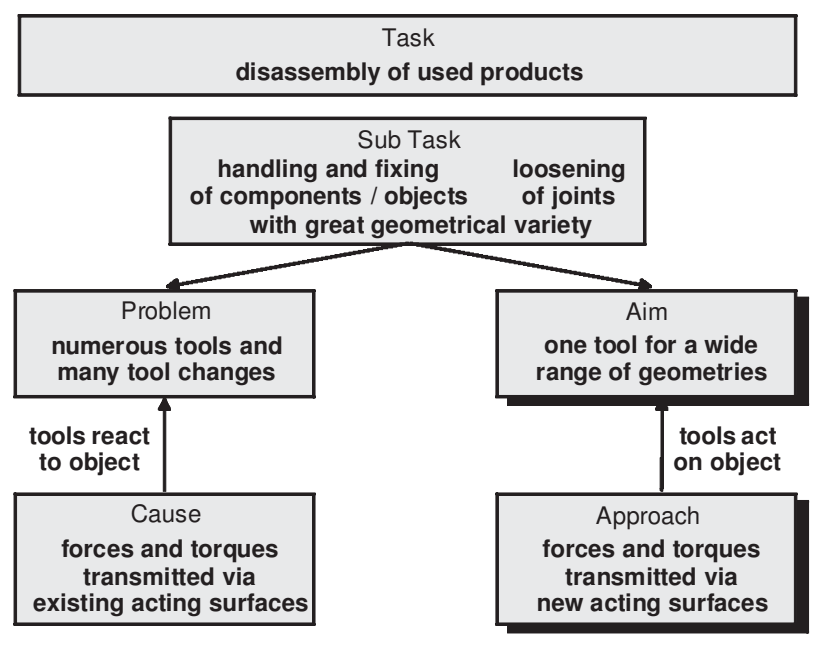

Fig. 1 General approach for disassembly tasks 
energy. This energy generates indents in the screw head [5]. For analysis of the indentation process, the following assumptions have been made for the generation of acting surface handling:

(a) wide independence from geometry and materials,

(b) low requirements for accessibility,

(c) fast generation of acting surfaces with small forces and torques,

(d) transmission of large forces and torques in all six degrees of freedom,

(e) minimization of sensor effort for positioning and orientation of objects and tools,

(f) avoiding damage of components behind the outer casing,

(g) manual and automated application.

According to these constraints and to experimental analysis, the roll threads process (indentation forming process with revolving motion) has been chosen. In contrast to DIN 8583, no core hole exists. Therefore, the process is better described as a combination of indentation and cutting with a geometrically defined edge. This process is similar to indentation mainly independent from material and geometry. The process enables fastacting surface generation and has low requirements for accessibility. Additionally, it is possible to transmit forces and torques in all six dimensions with the generated acting surface. In the following, two examples of disassembly tools will be presented that create their own acting surface on the disassembly object.

\section{GEOMETRY-INDEPENDENT DISASSEMBLY TOOL FOR LOOSENING SCREWS}

\subsection{Challenge}

The most well-known and also most frequently applied joining process is screwing. The wide range of wornout products and the great variety of joints within these products prevent an economic disassembly.

Up to now, ordinary screwing tools have been adapted to the existing geometry of screw heads by changes of the bit. Torques for loosening screws are transmitted through existing acting surfaces of the screw head. The amount of tools required, frequent tool changes and modifications caused by time and use, such as corrosion, dirt or varnish, make an economic screw disassembly more difficult. A geometry-independent disassembly screwer can reduce tool demand and the amount of capital employed.

The task was to develop a tool with shape-independent end-effectors for partly destructive disassembly. During the research, disjoining processes have been classified and worn-out products have been analysed. A mathematical model has been developed to describe the disjoining process as well as the techniques of the end-effector used to act upon the joining element.

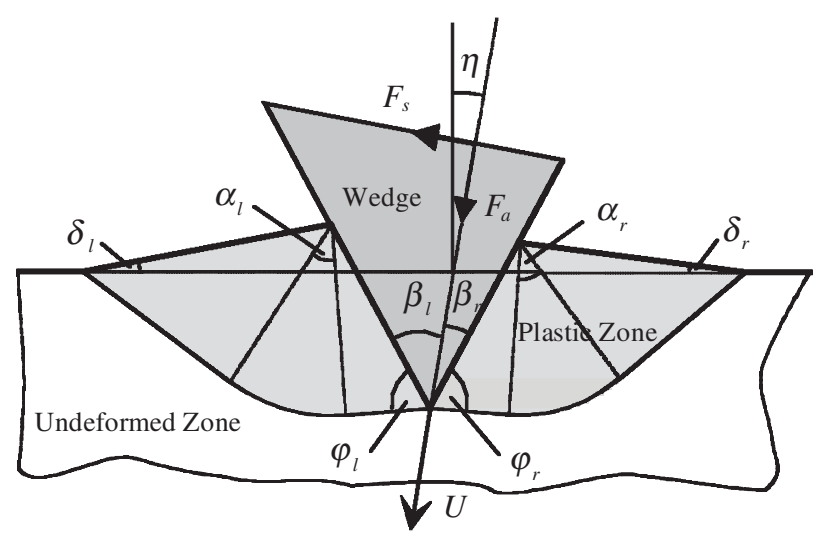

Fig. 2 Slip-line field for general wedge indentation

\subsection{Model}

\subsubsection{General wedge indentation}

The pursued approach is based on the following assumptions:

1. The indented material is semi-infinite and rigidperfectly plastic.

2. The wedge is rigid, straight sided and acute angled.

3. The angle of the axis of wedge inclined to the contact normal is situated inside the friction cone, i.e. $|\eta| \leqslant \tan ^{-1} \mu$, with $\eta>0$ implying right-side inclination. As the wedge is pressed into the material, a raised lip will be formed at each side of the wedge (see Fig. 2). The slip-line field is shown in Fig. 3.

From the yield condition [6], the normal and frictional stresses along AE can be expressed as

$$
\begin{aligned}
\sigma_{\mathrm{r}} & =p_{\mathrm{r}}+k \sin 2 \varphi_{\mathrm{r}} \\
\tau_{\mathrm{r}} & =-k \cos 2 \varphi_{\mathrm{r}}
\end{aligned}
$$

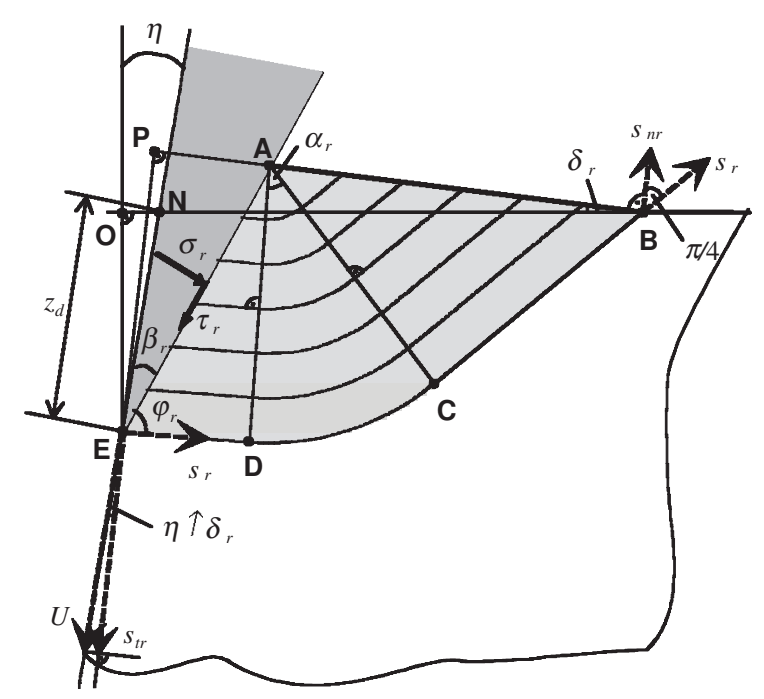

Fig. 3 Slip-line field on the right side 
Hencky's theorem gives

$$
p_{\mathrm{r}}=k\left(1+2 \alpha_{\mathrm{r}}\right)
$$

and therefore

$$
\sigma_{\mathrm{r}}=k\left(1+2 \alpha_{\mathrm{r}}+\sin 2 \varphi_{\mathrm{r}}\right)
$$

The friction constraint on the contact interface yields

$$
\tau_{\mathrm{r}}=\mu \sigma_{\mathrm{r}}
$$

Substituting (2) and (4) into (5) gives

$$
-\cos 2 \varphi_{\mathrm{r}}=\mu\left(1+2 \alpha_{\mathrm{r}}+\sin 2 \varphi_{\mathrm{r}}\right)
$$

From Fig. 3, the following geometric relation is obvious:

$$
\delta_{\mathrm{r}}=\beta_{\mathrm{r}}+\eta-\alpha_{\mathrm{r}}+\varphi_{\mathrm{r}}-\frac{\pi}{4}
$$

The cinematic constraint during the indentation yields

$$
\begin{aligned}
& 2 \sin ^{2} \varphi_{\mathrm{r}} \sin \delta_{\mathrm{r}} \cos \left(\delta_{\mathrm{r}}-\eta\right)+2 \sqrt{2} \sin \varphi_{\mathrm{r}} \sin \beta_{\mathrm{r}} \sin \delta_{\mathrm{r}} \\
& \quad-\sin \beta_{\mathrm{r}} \cos \left(\beta_{\mathrm{r}}+\eta\right)=0
\end{aligned}
$$

Further details of equation (8) can be found in the Appendix.

It is noted that the parameters $\varphi_{\mathrm{r}}, \alpha_{\mathrm{r}}$ and $\delta_{\mathrm{r}}$ can be solved from equations (6) to (8). Accordingly, the normal stress $\sigma_{\mathrm{r}}$ and frictional stress $\tau_{\mathrm{r}}$ are uniquely determined.

On the left side of the wedge, equations (7) and (8) are replaced by

$$
\begin{aligned}
& \delta_{1}=\beta_{1}-\eta-\alpha_{1}+\varphi_{1}-\frac{\pi}{4} \\
& 2 \sin ^{2} \varphi_{1} \sin \delta_{1} \cos \left(\delta_{1}+\eta\right)+2 \sqrt{2} \sin \varphi_{1} \sin \beta_{1} \sin \delta_{1} \\
& \quad-\sin \beta_{1} \cos \left(\beta_{1}-\eta\right)=0
\end{aligned}
$$

For details of equation (10), see the Appendix.

The result is that the geometric and cinematic constraints are not identical on both sides of the wedge. This leads to asymmetrical stress distribution along the contact interfaces.

The friction constraint and yield condition on the left side are similarly expressed as

$$
\begin{aligned}
& -\cos 2 \varphi_{1}=\mu\left(1+2 \alpha_{1}+\sin 2 \varphi_{1}\right) \\
& \sigma_{1}=k\left(1+2 \alpha_{1}+\sin 2 \varphi_{1}\right) \\
& \tau_{1}=-k \cos 2 \varphi_{1}
\end{aligned}
$$

Based on equations (9) to (13), all the parameters on the left side are determined. Consequently, the axial and side indentation forces (Fig. 2) are given by

$$
\begin{aligned}
F_{\mathrm{a}}=b_{\mathrm{w}} z_{\mathrm{d}} \cos \eta & {\left[\frac{\sigma_{\mathrm{r}} \sin \beta_{\mathrm{r}}+\tau_{\mathrm{r}} \cos \beta_{\mathrm{r}}}{\cos \left(\beta_{\mathrm{r}}+\eta\right)-\sqrt{2} \sin \varphi_{\mathrm{r}} \sin \delta_{\mathrm{r}}}\right.} \\
& \left.+\frac{\sigma_{1} \sin \beta_{1}+\tau_{1} \cos \beta_{1}}{\cos \left(\beta_{1}-\eta\right)-\sqrt{2} \sin \varphi_{1} \sin \delta_{1}}\right]
\end{aligned}
$$

$$
\begin{aligned}
F_{\mathrm{s}}=b_{\mathrm{w}} z_{\mathrm{d}} \cos \eta & {\left[\frac{-\sigma_{\mathrm{r}} \sin \beta_{\mathrm{r}}+\tau_{\mathrm{r}} \cos \beta_{\mathrm{r}}}{\cos \left(\beta_{\mathrm{r}}+\eta\right)-\sqrt{2} \sin \varphi_{\mathrm{r}} \sin \delta_{\mathrm{r}}}\right.} \\
& \left.+\frac{\sigma_{1} \sin \beta_{1}-\tau_{1} \cos \beta_{1}}{\cos \left(\beta_{1}-\eta\right)-\sqrt{2} \sin \varphi_{1} \sin \delta_{1}}\right]
\end{aligned}
$$

Some details on contact length are presented in the Appendix.

When $\beta_{\mathrm{r}}=\beta_{1}=\beta$ and $\eta=0$, the contact stress distribution becomes symmetrical. In this case, the side force disappears and only the axial force is retained:

$$
\begin{aligned}
& F_{\mathrm{s}}=0 \\
& F_{\mathrm{a}}=\frac{2(\sigma \sin \beta+\tau \cos \beta) b_{\mathrm{w}} z_{\mathrm{d}}}{\cos \beta-\sqrt{2} \sin \varphi \sin \delta}
\end{aligned}
$$

It can be seen that the indentation force increases linearly with indentation depth.

\subsubsection{Perfectly rough indentation}

When the coefficient of friction $\mu$ reaches a certain value, shearing along the sides of the wedge occurs. This is the so-called perfectly rough indentation. In this case, the frictional stress equals the shear yield stress $k$ on the side where shearing occurs. Two possible slip-line fields are shown in Fig. 4.

The limit coefficients of friction corresponding to shearing, $\mu_{\mathrm{r}}^{*}$ and $\mu_{1}^{*}$, are determined by

$$
\varphi_{\mathrm{r}}=\pi / 2 \quad \text { and } \quad \varphi_{1}=\pi / 2
$$

respectively. For $\eta=0,5$ and $10^{\circ}$, the values of $\mu_{\mathrm{r}}^{*}$ and $\mu_{1}^{*}$ for various semi-wedge angles are shown in Fig. 5. From
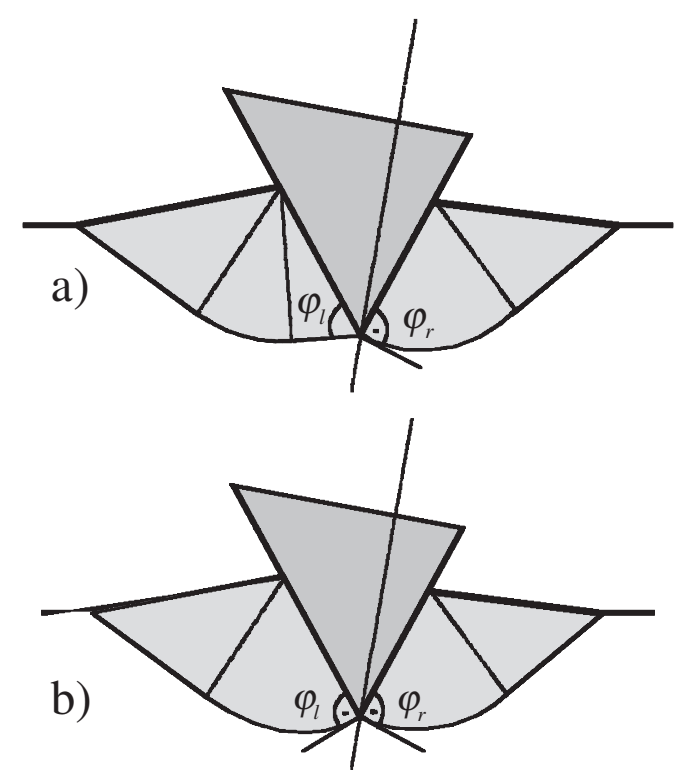

Fig. 4 Two possible slip-line fields for perfectly rough indentation: (a) shearing on one side $\left(\varphi_{1}<90^{\circ}, \varphi_{\mathrm{r}}=90^{\circ}\right)$; (b) shearing on both sides of the wedge $\left(\varphi_{1}=90^{\circ}\right.$, $\varphi_{\mathrm{r}}=90^{\circ}$ ) 


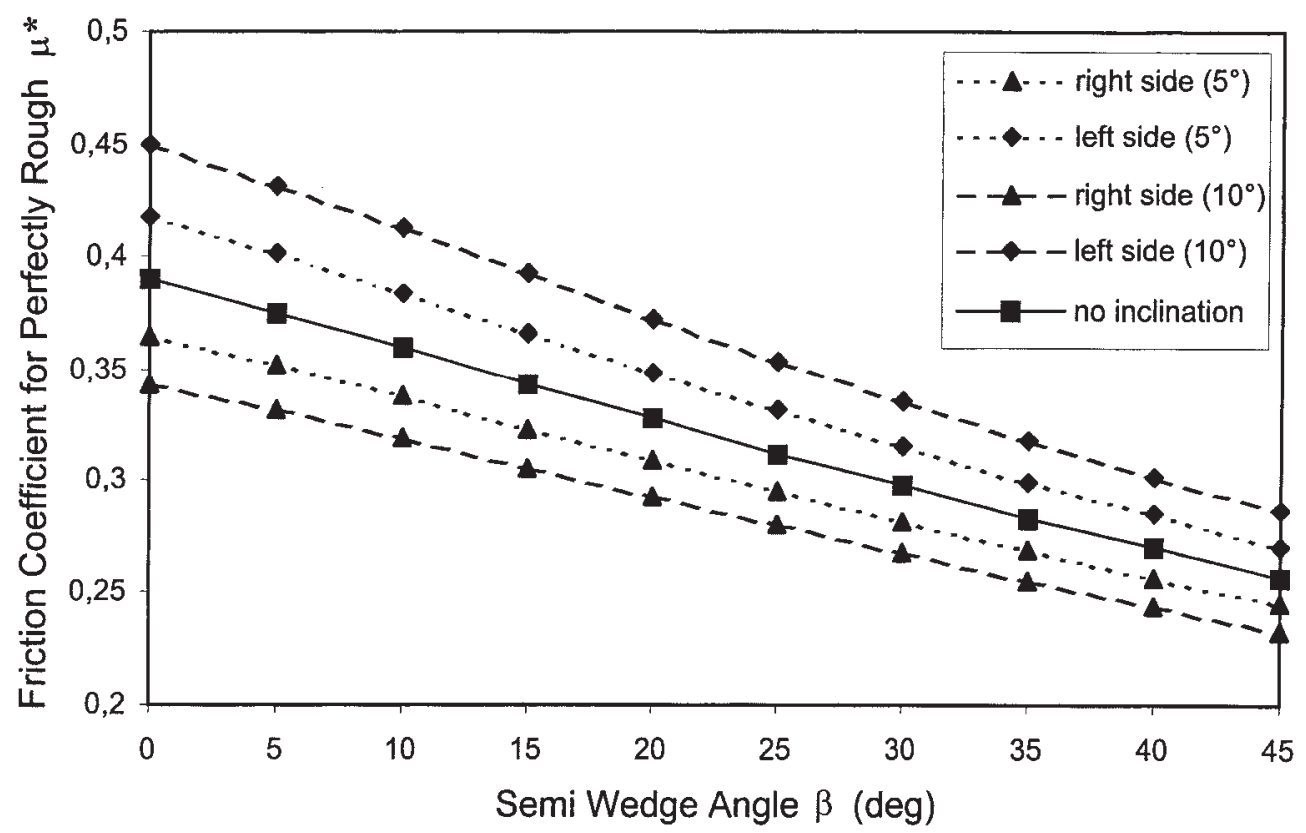

Fig. 5 Coefficient of friction for perfectly rough indentation at different inclined angles

Fig. 5 it can be concluded that:

1. The coefficient of friction for perfectly rough $\mu^{*}$ decreases with increase in the semi-wedge angle $\beta$.

2. For symmetrical indentation $\left(\eta=0\right.$ and $\left.\beta_{\mathrm{r}}=\beta_{1}\right)$, $\mu_{\mathrm{r}}^{*}=\mu_{1}^{*}$, and shearing always sets in simultaneously on both sides of the wedge with increase in $\mu$.

3. For asymmetrical indentation $\left(\eta \neq 0\right.$ or $\left.\beta_{\mathrm{r}} \neq \beta_{1}\right)$, generally $\mu_{\mathrm{r}}^{*} \neq \mu_{1}^{*}$. With increase in $\mu$, shearing sets in first on one side of the wedge (Fig. 4a) and then on both sides of the wedge (Fig. 4b). In the latter case, $\mu \geqslant \max \left\{\mu_{1}^{*}, \mu_{\mathrm{r}}^{*}\right\}$, and the contact stresses on both sides are equal. However, the side indentation force $F_{\mathrm{s}}$ may still be retained owing to the inequality of contact length.

4. For indentation where the shape of the wedge is symmetrical $\left(\beta_{\mathrm{r}}=\beta_{1}\right)$, the difference between $\mu_{\mathrm{r}}^{*}$ and $\mu_{1}^{*}$ increases with increase in $\eta$. This implies that $\eta$ can be regarded as a measure of the extent of asymmetrical stress distribution.

\subsection{Verification}

Some measurements are presented to verify the improved model [7]. Wedges with different semi-angles $(\beta=20,30$ and $40^{\circ}$, fully hardened high-speed cutting alloy EW9Co10) were pressed into cubic samples made of AlMgSi0.5F22. Force and displacement sensors are used to measure the indentation force and depth. The shear yield stress for the indented material is $k=138 \mathrm{~N} / \mathrm{mm}^{2}$, and the coefficient of friction between the wedge and the indented material is $\mu=0.35$.

It must be noted that the present $\mu$ exceeds the limit value for perfectly rough indentation. For $\beta=20,30$ and $40^{\circ}$, the coefficients of friction for perfectly rough indentation are $\mu^{*}=0.3278,0.2974$ and 0.2697 respectively (Fig. 5). Thus, shearing occurs on both sides of the wedge. The indentation force and depth for different semi-wedge angles are shown in Fig. 6. It can be seen that the theoretical results have a good match with experimental ones. The indentation force approximately maintains a linear relation with the indentation depth. This becomes more obvious for deeper indentation.

\subsection{Prototypical tool}

Based on the analytical and experimental results of the indention process, a flexible tool for the loosening of screws has been developed to test the acting surface generation approach. Based on the developed process model, the necessary acting surface size and the indention depth for transmitting the loosening torque can be determined. Thus, the necessary impact energy can be calculated. From the impact energy the geometrical dimensions of the cylinder can be designed. For the loosening of screws (e.g. hexagon head cap screw M8, DIN 933) over a generated acting surface, an indention depth of $0.9 \mathrm{~mm}$ will be needed. Therefore, a deformation energy of $6.12 \mathrm{~J}$ will be necessary. With screws of higher stability, higher deformation energies will be needed.

The prototype of this tool generates the new acting surfaces by using an pneumatically driven internal impact mass with minimized reaction forces for the users (Fig. 7). The operation mode of the disassembly tool is shown in Fig. 8.

The necessary acting surface is generated by a highfrequency impact mechanism with a maximum energy of 


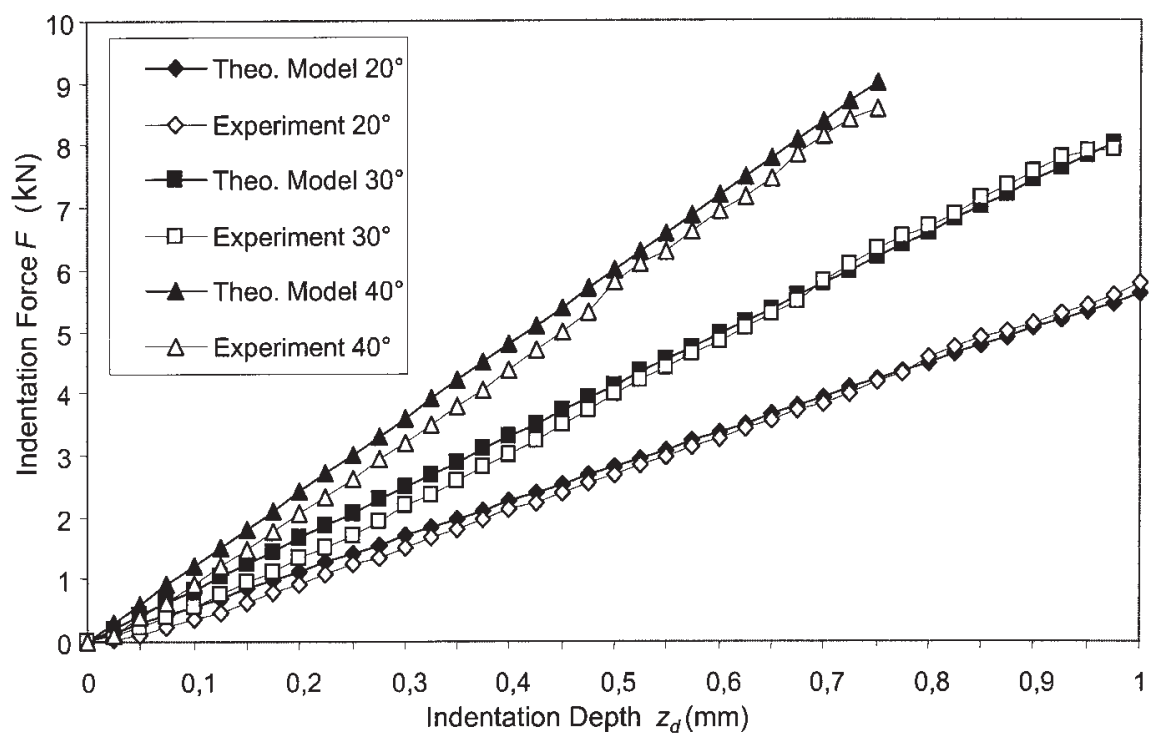

Fig. 6 Indentation force and depth for different semi-wedge angles

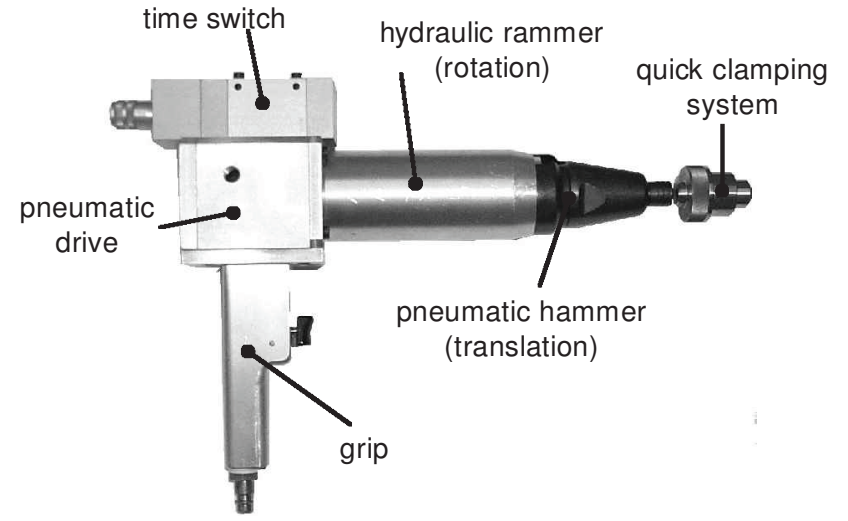

Fig. 7 Prototypical screw disassembly tool
$15 \mathrm{~J}$ and a frequency of $50 \mathrm{~Hz}$. The duration of the impact can be adjusted with a time switch by the worker. The necessary duration depends on the size and hardness of the screw. After generating the acting surface, the screw is unscrewed by a pneumatic drive. A hydraulic rammer supports the unscrewing process with tight screws by transferring rotatory impacts. Conventional bits can also be inserted in the high-speed clamping system instead of the sharp-edged end-effectors. Thus, the tool can be used like a normal compressed-air screwdriver. For simple and safe application, a special centring device has been developed, which enables the worker to centre the end-effector safely on the screw head. The device is partly size and geometry independent.

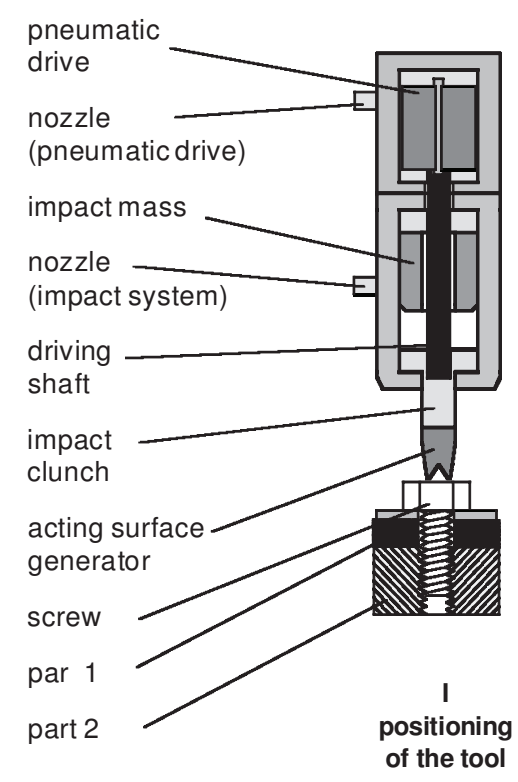

compressed air
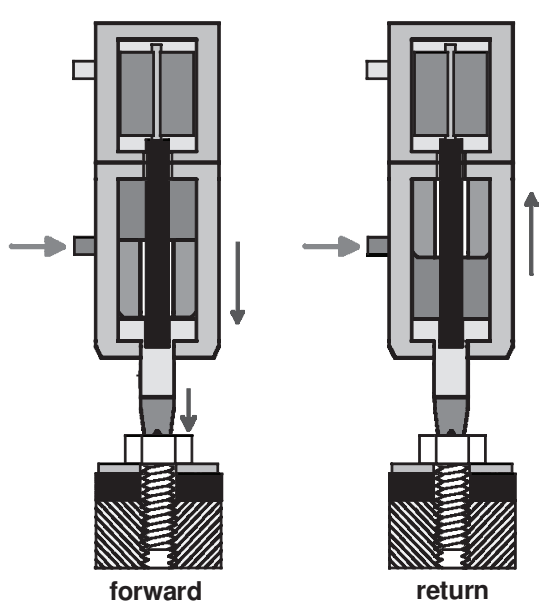

II

high frequency impacts

with acting

surface generation

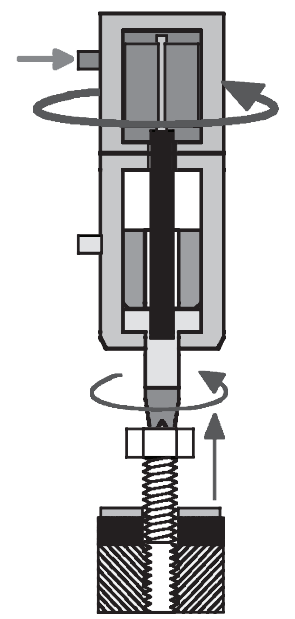

III

unscrewing

Fig. 8 Operation sequence of the prototype tool for the screw disassembly of screw joints 


\section{FLEXIBLE TOOL FOR HANDLING OPERATIONS}

\subsection{Challenge}

It is noted that the objective of disassembly is to regain reusable components and to generate specific material fractions compatible with the respective recycling processes. Therefore, many handling operations, especially the removal of housings fitted with loose connections such as snaps, clips, light press fits, free-running fits, etc., are necessary. For the disassembly of loose connections, reliable handling is more important than accurate position sensing. With the joining together of tool and object, a strong form closure is prepared so as to transmit forces and torques. During the joining process, the handled object may or may not be destroyed.

Up to now, handling has mainly been done manually. Large components, dirt, heavy weight and also sharp edges hamper manual handling. The challenge is to develop flexible tools for quick and safe handling of plastic casings. In the following, a mathematical model for the calculation of the torque for a screw-like endeffector penetrating a plastic case will be described.

\subsection{Model}

To perform an upper bound analysis, a cinematically admissible [8] velocity field must be available beforehand. Here, the plastic material is supposed to move upwards along a series of helical surfaces parallel to the groove surfaces of the screwnail. The screwnail has been chosen as a reference coordinate system. For any point $\mathrm{P}$ in the plastic region of the screwnail head, its absolute velocity $v$ can be regarded as the resultant of the reference velocity $U$ and the relative velocity $w$ (see Fig. 9). The former is referred to the absolute velocity of the screwnail, while the latter describes the relative motion between the plastic material and the screwnail.

For a point on the boundary, the relative motion occurs only along the tangential plane of the screw interface. This means that the relative velocity can be further decomposed into two components: one along the tangent direction of a spiral winding around a cone, the other along the outer radial direction (see Fig. 9). Based on the boundary conditions and the condition of material incompressibility, the velocity field is given as follows:

Velocity of the screwnail:

$$
\left[\begin{array}{l}
u_{r} \\
u_{\varphi} \\
u_{z}
\end{array}\right]=\left[\begin{array}{c}
0 \\
-\frac{r}{b_{\mathrm{s}}} \\
-1
\end{array}\right] U, \quad \text { where } b_{\mathrm{s}}=p /(2 \pi)
$$

Velocity of the plastic material in the plastic region of the screwnail head:

$$
\begin{aligned}
& {\left[\begin{array}{c}
v_{r} \\
v_{\varphi} \\
v_{z}
\end{array}\right]=\left[\begin{array}{c}
\lambda_{3} \frac{z}{r}+\lambda_{4} \\
\left(\lambda_{1} \frac{z}{r}+\lambda_{2}\right) \frac{r}{b_{\mathrm{s}}} \\
\lambda_{1} \frac{z}{r}+\lambda_{2}
\end{array}\right] U,} \\
& {\left[\begin{array}{c}
w_{r} \\
w_{\varphi} \\
w_{z}
\end{array}\right]=\left[\begin{array}{c}
\lambda_{3} \frac{z}{r}+\lambda_{4} \\
\left(\lambda_{1} \frac{z}{r}+\lambda_{2}+1\right) \frac{r}{b_{\mathrm{s}}} \\
\lambda_{1} \frac{z}{r}+\lambda_{2}+1
\end{array}\right] U}
\end{aligned}
$$

Velocity of the plastic material in the plastic region of the screwnail body:

$$
\begin{aligned}
& {\left[\begin{array}{c}
v_{r} \\
v_{\varphi} \\
v_{z}
\end{array}\right]=\left[\begin{array}{c}
\left(\lambda_{1} \frac{h_{0}}{r}+\lambda_{2}\right) \frac{r}{b_{\mathrm{s}}} \\
\lambda_{1} \frac{h_{0}}{r}+\lambda_{2}
\end{array}\right] U,} \\
& 0 \\
& {\left[\begin{array}{c}
w_{r} \\
w_{\varphi} \\
w_{z}
\end{array}\right]=\left[\begin{array}{c}
\left(\lambda_{1} \frac{h_{0}}{r}+\lambda_{2}+1\right) \frac{r}{b_{\mathrm{s}}} \\
\lambda_{1} \frac{h_{0}}{r}+\lambda_{2}+1
\end{array}\right] U}
\end{aligned}
$$

where $\lambda_{i}(i=1, \ldots, 4)$ are the velocity coefficients determined by boundary conditions and the condition of material incompressibility.

\subsubsection{Upper bound of indentation torque}

The upper bound formulation [9] is given by

$$
\dot{E}=\sqrt{2} k \int_{V} \sqrt{\dot{\varepsilon}_{i j} \dot{\varepsilon}_{i j}} \mathrm{~d} V+\int_{S_{V}} \tau|\Delta v| \mathrm{d} S-\int_{S_{T}} T_{i} v_{i} \mathrm{~d} S
$$

where $\dot{E}$ denotes the total rate of energy dissipation for the deforming workpiece, and $k$ is the shear yield stress. The first term represents the rate of energy dissipated during plastic flow. The second term accounts for the energy dissipated in overcoming friction on the external surfaces and the energy dissipated along the internal surfaces of the velocity discontinuity. The last term is included to account for the effects of applied traction and is zero for the process being considered here. In detail, the total rate of energy dissipation, $\dot{E}$, consists of nine parts:

$$
\begin{aligned}
\dot{E}= & \dot{E}_{\mathrm{H}}+\dot{E}_{\mathrm{B}}+\dot{E}_{\mathrm{S}}+\dot{E}_{\mathrm{HO}}+\dot{E}_{\mathrm{BO}}+\dot{E}_{\mathrm{HI}}+\dot{E}_{\mathrm{BI}} \\
& +\dot{E}_{\mathrm{HS}}+\dot{E}_{\mathrm{BS}}
\end{aligned}
$$



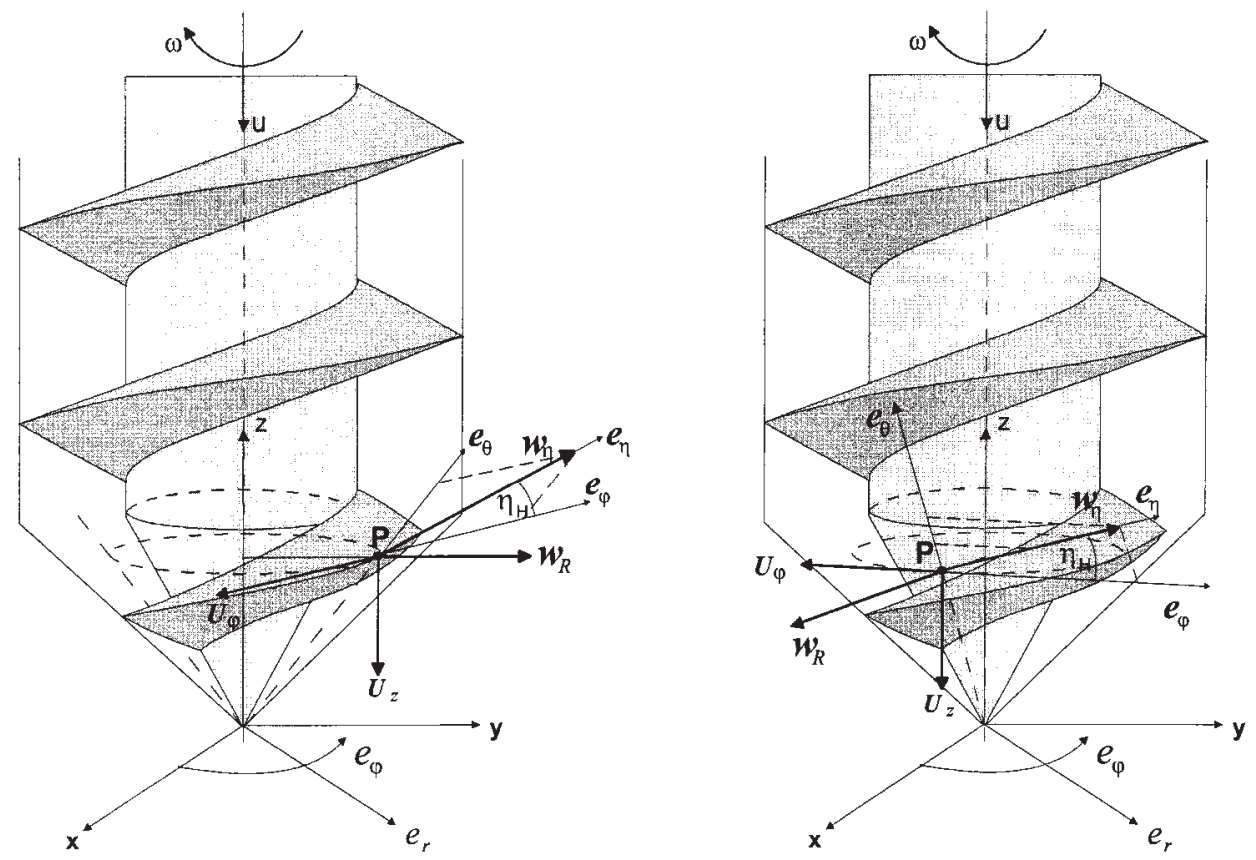

Fig. 9 Velocity of a point on the boundary

where $\dot{E}_{\mathrm{H}}$ and $\dot{E}_{\mathrm{B}}$ are the rates of energy dissipated owing to plastic flow in the grooves of the screwnail head and body respectively, $\dot{E}_{\mathrm{S}}, \dot{E}_{\mathrm{HO}}$ and $\dot{E}_{\mathrm{BO}}$ are the rates of energy dissipated owing to the velocity discontinuity along the ring-like plane separating the screwnail head from the body, the outer cone surface of the screwnail head and the outer cylindrical surface of the screwnail body respectively, and $\dot{E}_{\mathrm{HI}}, \dot{E}_{\mathrm{BI}}, \dot{E}_{\mathrm{HS}}$ and $\dot{E}_{\mathrm{BS}}$ are the rates of energy dissipated owing to friction along the inner cone surface and screw interface of the screwnail head, the inner cylindrical surface and the screw interface of the screwnail body respectively. Figure 10 shows the involved geometric surfaces.

The total rate of energy dissipation can be expressed as

$$
\dot{E}=k U \sum_{x} m_{x}
$$

where $m_{x}(x=\mathrm{H}, \mathrm{B}, \mathrm{S}, \mathrm{HO}, \mathrm{BO}, \mathrm{HI}, \mathrm{BI}, \mathrm{HS}, \mathrm{BS})$ are the energy coefficients, $\dot{E}_{x}=k U m_{x}$.

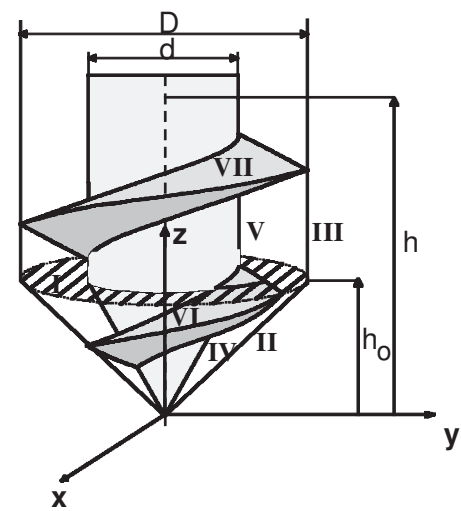

Note that the work done by the indentation torque is

$$
\dot{E}=T \frac{U}{b_{\mathrm{s}}}
$$

The upper bound of the indentation torque is eventually given by

$$
T=k b_{\mathrm{s}} \sum_{x} m_{x}
$$

It can be seen that the indentation torque is a function of shear yield stress, screwnail geometry and indentation depth. An increment in indentation depth requires a linear increment in input torque. The torque can be reduced by optimizing the geometry of the screwnails. This is a topic for future work.

\subsection{Verification}

For experimental set-up, the designed disassembly tool is

\footnotetext{
I.... Ring-like plane separating screwnail head from body

II.... Outer cone surface of screwnail head

III.... Outer cylindrical surface of screwnail body $(\mathrm{BO})$

IV.... Inner cone surface of screwnail head $(\mathrm{HI})$

V.... Inner cylindrical surface of screwnail body (BI)

VI.... Groove interface of screwnail head (HS)

VII.... Groove interface of screwnail body (BS)
}

Fig. 10 Internal surfaces with a velocity discontinuity (I-III) and external surfaces with friction (IV-VII) 


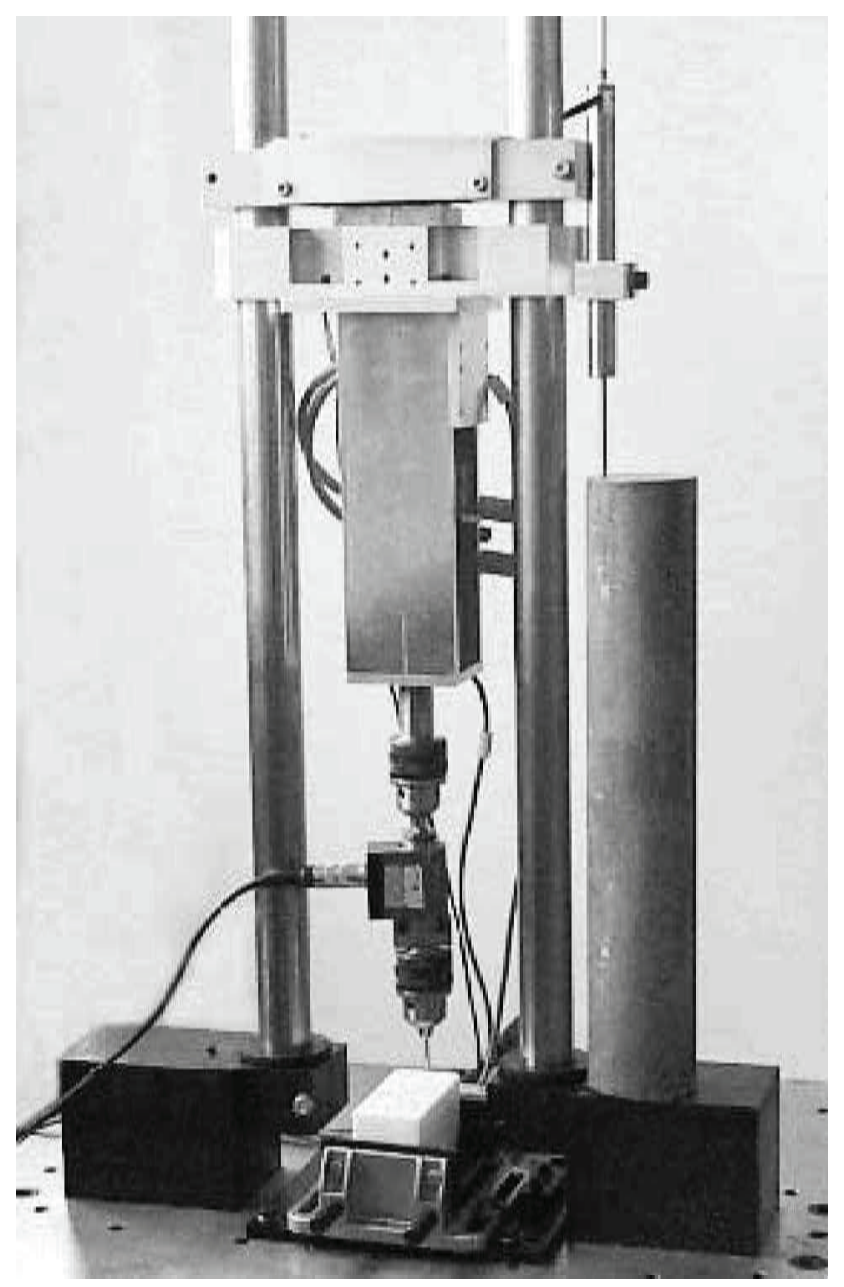

Fig. 11 Experimental set-up

connected to two sensory systems in order to measure the indentation torque and the indentation depth (see Fig. 11). The torque sensor is from Newport Omega Electronics (type TQ503-63), with a range of 0-63 N m and an accuracy of 0.002 per cent. The data from the torque sensor is transferred to a type IFNS 1000-1/E
Newport panel meter and then to a PC. The indentation depth is measured by an NT25 Megatron contact distance sensor. The experimental data are stored in the PC. The experiments are carried out with different screws and plastics. One is described as follows:

Screw type 1

Maximum diameter $\quad 5 \mathrm{~mm}$

Minimum diameter $\quad 3 \mathrm{~mm}$

Height of the screwnail head $5 \mathrm{~mm}$

Material 1

Polyethylene

Shear yield stress $\quad k=15.25 \mathrm{~N} / \mathrm{mm}^{2}$

It can be seen that there is a good match between the predicted values for the indentation torque and the measurements (Fig. 12). The measured values have an offset because of the upper bound method used. However, the difference is small enough to estimate the required indentation torque exerted by the driving unit of the disassembly tool. Furthermore, it can be used to estimate the reaction forces and torques to the robot in automatic disassembly or to the worker in manual use.

\subsection{Prototypical tool}

Figure 13 shows a prototype gripper for plastic material recycling. The handled object is the plastic frame of a computer monitor, where there exist screw connections as well as snap connections. Suppose the former were separated beforehand by certain unscrewing tools (e.g. references [5] and [7]). The task here is to separate the latter. A screwnail is integrated into the end-effector (see Fig. 13). Modified screws are used as end-effectors for plastic, whereas for metal combined tap drills are used. With a drilling process, the prototypical gripper can create a new acting surface with the plastic frame, so that a strong form closure is prepared for the next disassembly operations. The completion of self-connection

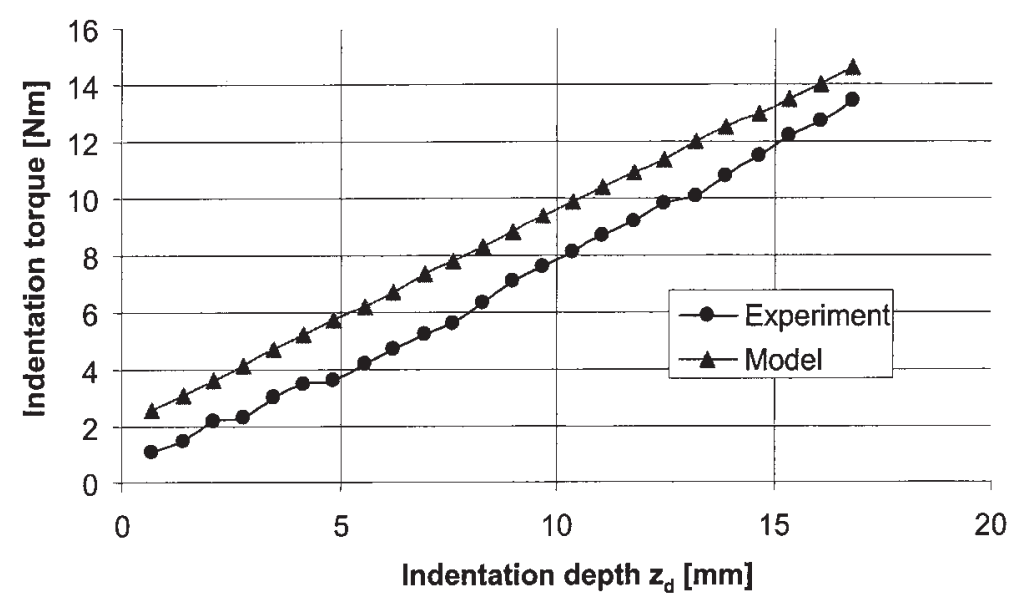

Fig. 12 Indentation torque versus indentation depth for screw type 1 and material 1 


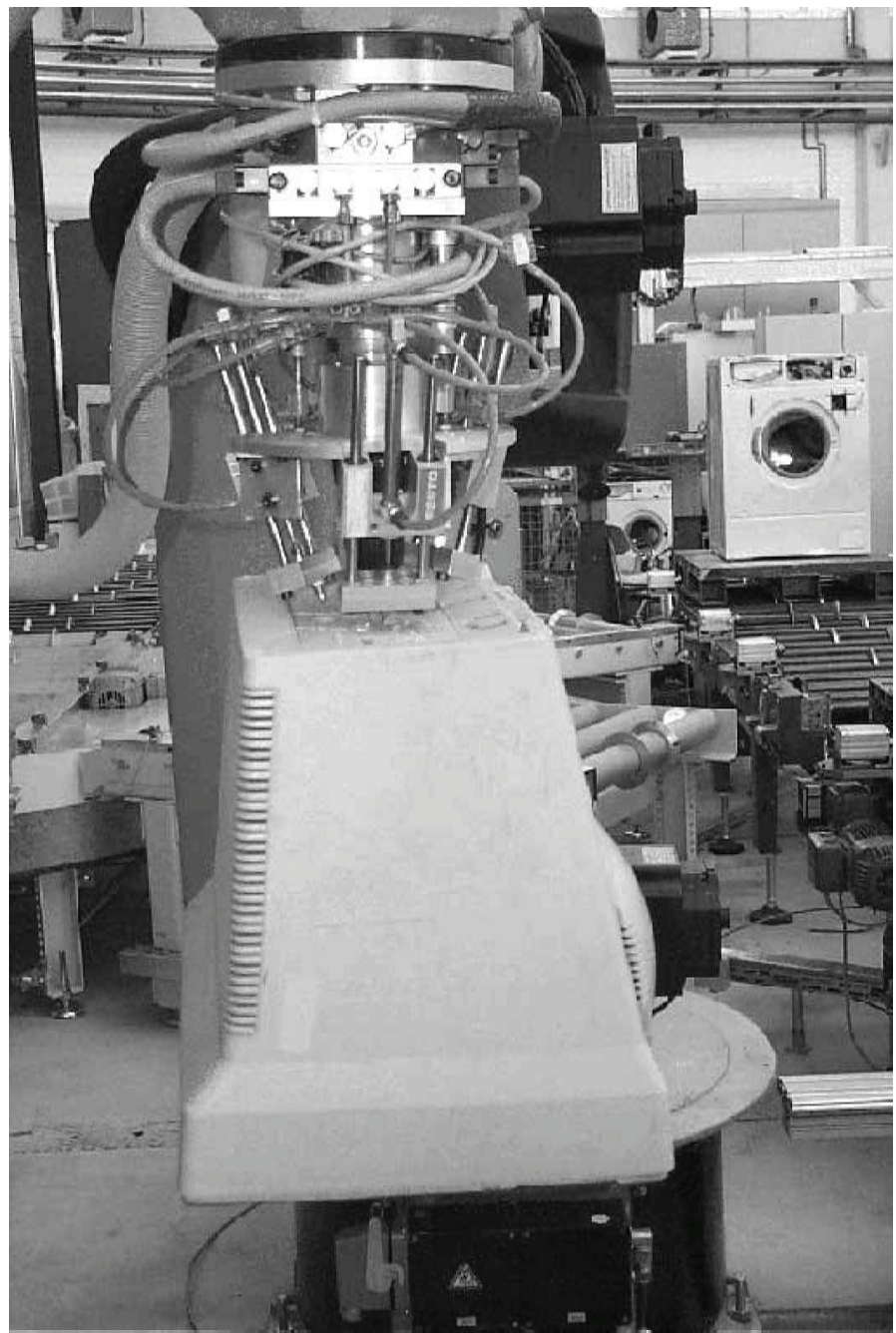

Fig. 13 Prototypical gripper for plastic material recycling

does not require accurate position sensing of the snap connections, and drilling of the screwnail can overcome the geometry uncertainties of object surfaces. The approximate value for the position is detected by a vision system or manually by a worker.

When all the snap connections are loosened, the screwnail will be taken out of the plastic material by an inverse drilling process. In both the self-connection and separation processes, integrated needles play the role of fixing. Once the drive pressure reduces to zero, the needles automatically separate the plastic frame. All the advantages of this gripper are achieved at the cost of object destruction. Thus, the gripper is specially oriented to material recycling.

\section{SUMMARY AND DIRECTIONS FOR FURTHER RESEARCH}

One of the most intriguing aspects of disassembly is that it has to deal with products not designed for recycling.
This paper presents an approach to support the design of flexible disassembly tools: the joining together of tool and object, leading to a strong form closure to transmit forces and torques. The disassembly task with the generating new acting surface approach is decomposed into three processes:

(a) acting surface generation,

(b) task execution,

(c) separation.

Future work may include more experiments on the prototype tools, the development of algorithms for planning disassembly operations and the application of the new approach to other industrial fields. A modular kit for disassembly will be the focus of further research. With the kit, tools for manual and automated loosening and handling should be realized. Suitable modules should be chosen and constructed. The tools should be applicable as stationary and mobile. For cost reduction purposes the modules should also be used for assembly. 


\section{ACKNOWLEDGEMENT}

This paper presents results of Special Research Programme 281 'Disassembly Factories', financially supported by the Deutsche Forschungsgemeinschaft (DFG).

\section{REFERENCES}

1 Hentschel, C., Seliger, G. and Zussman, E. Grouping of used products for cellular recycling systems. Ann. CIRP, 1995, 44(1), 11-14.

2 Seliger, G., Hentschel, C. and Wagner, M. Disassembly factories for recovery of resources in product and material cycles. In Life Cycle Modelling for Innovative Products and Processes, 1996 (Chapman and Hall, London).

3 DIN 8583 Blatt 5. Fertigungsverfahren Druckumformen; Eindrücken; Unterteilung, Begriffe, 1970 (Beuth Verlag, Berlin).

4 DIN 8580. Fertigungsverfahren: Begriffe, Einteilungen, 1985 (Beuth Verlag, Berlin).

5 Seliger, G. and Rebafka, U. Transfer project E1 to Special Research Programme 281 'Disassembly Factories': tools for forming and loosening of connections. Working Report, Transfer project E1, 1999.

6 Hill, H. Mathematical Theory of Plasticity, 1956 (Oxford University Press).

7 Wagner, M. and Seliger, G. Modelling of geometryindependent endeffectors for flexible disassembly tools. In Proceedings of 3rd International CIRP Seminar on Life Cycle Engineering (Eco-performance '96), Zürich, 1996, pp. 219-228 (Verlag Industrielle Organisation).

8 Prager, W. and Hodge, P. G. Theory of Perfectly Plastic Solids, 1951 (John Wiley).

9 Seliger, G., Zuo, B. R. and Stenzel, A. Determination of indentation forces for disassembly process. In Proceedings of 1999 IEEE International Symposium on Assembly and Task Planning (ISATP '99), Porto, Portugal, 21-24 July 1999, pp. 141-145.

10 Hill, R., Lee, E. H. and Tupper, S. J. The theory of wedge indentation of ductile materials. Proc. R. Soc. (Lond.) A, 1947, 188, 273-289.

\section{APPENDIX}

\section{Cinematic constraints for general wedge indentation}

Consider the right side of the wedge. The following derivation is similar to that in reference [10]. The notation refers to Fig. 3.

Along AE, the velocity of the wedge and that of the plastic material must have the same projection on the normal to AE. Thus, at every point the plastic material has the velocity

$$
s_{\mathrm{r}}=v \frac{\sin \beta_{\mathrm{r}}}{\sin \varphi_{\mathrm{r}}}
$$

The lip surface $\mathrm{AB}$ undergoes a translation in the direction $\mathrm{CB}$ with the velocity $v_{r}$, whose component perpendicular to $\mathrm{AB}$ is given by

$$
s_{\mathrm{n} r}=v \frac{\sin \beta_{\mathrm{r}}}{\sqrt{2} \sin \varphi_{\mathrm{r}}}
$$

At the same time, the velocity component of the wedge tip in the opposite direction is

$$
s_{\mathrm{tr}}=v \cos \left(\delta_{\mathrm{r}}-\eta\right)
$$

Therefore, the distance of $\mathrm{E}$ from $\mathrm{AB}$ can be expressed as

$$
\mathrm{PE}=z_{\mathrm{d}}\left[\frac{\sin \beta_{\mathrm{r}}}{\sqrt{2} \sin \varphi_{\mathrm{r}}}+\cos \left(\delta_{\mathrm{r}}-\eta\right)\right]
$$

where $z$ is the indentation depth.

From Fig. 3, the following relations are obvious:

$$
\begin{aligned}
& \mathrm{AE}=\frac{\mathrm{AB}}{\sqrt{2} \sin \varphi_{\mathrm{r}}} \\
& \mathrm{AE} \cos \left(\beta_{\mathrm{r}}+\eta\right)-\mathrm{AB} \sin \delta_{\mathrm{r}}=\mathrm{OE}
\end{aligned}
$$

Note that

$$
\mathrm{OE}=z_{\mathrm{d}} \cos \eta
$$

From equations (31) to (33), the contact length and lip length are given by

$$
\begin{aligned}
& L_{\mathrm{rcontact}}=\mathrm{AE}=\frac{z_{\mathrm{d}} \cos \eta}{\cos \left(\beta_{\mathrm{r}}+\eta\right)-\sqrt{2} \sin \varphi_{\mathrm{r}} \sin \delta_{\mathrm{r}}} \\
& L_{\mathrm{r} \text { lip }}=\mathrm{AB}=\frac{z_{\mathrm{d}} \cos \eta \sqrt{2} \sin \varphi_{\mathrm{r}}}{\cos \left(\beta_{\mathrm{r}}+\eta\right)-\sqrt{2} \sin \varphi_{\mathrm{r}} \sin \delta_{\mathrm{r}}}
\end{aligned}
$$

Also note that

$$
\begin{aligned}
& \mathrm{AE} \sin \left(\beta_{\mathrm{r}}+\eta\right)+\mathrm{AB} \cos \delta_{\mathrm{r}}=\mathrm{OB} \\
& \mathrm{OB} \sin \delta_{\mathrm{r}}+\mathrm{OE} \cos \delta_{\mathrm{r}}=\mathrm{PE}
\end{aligned}
$$

Substituting equations (30) and (33) to (35) into equations (36) and (37) yields

$$
\begin{aligned}
& 2 \sin ^{2} \varphi_{\mathrm{r}} \sin \delta_{\mathrm{r}} \cos \left(\delta_{\mathrm{r}}-\eta\right)+2 \sqrt{2} \sin \varphi_{\mathrm{r}} \sin \beta_{\mathrm{r}} \sin \delta_{\mathrm{r}} \\
& \quad-\sin \beta_{\mathrm{r}} \cos \left(\beta_{\mathrm{r}}+\eta\right)=0
\end{aligned}
$$

Equation (38) describes the cinematic constraint on the right side of the wedge.

Similarly, on the left side it follows that

$$
\begin{aligned}
& L_{1 \text { contact }}=\frac{z_{\mathrm{d}} \cos \eta}{\cos \left(\beta_{1}-\eta\right)-\sqrt{2} \sin \varphi_{1} \sin \delta_{1}} \\
& L_{1 \text { lip }}=\frac{z_{\mathrm{d}} \cos \eta \sqrt{2} \sin \varphi_{1}}{\cos \left(\beta_{1}-\eta\right)-\sqrt{2} \sin \varphi_{1} \sin \delta_{1}} \\
& 2 \sin ^{2} \varphi_{1} \sin \delta_{1} \cos \left(\delta_{1}+\eta\right)+2 \sqrt{2} \sin \varphi \sin \beta_{1} \sin \delta_{1} \\
& \quad-\sin \beta_{1} \cos \left(\beta_{1}-\eta\right)=0
\end{aligned}
$$


Equation (41) describes the cinematic constraint on the left side of the wedge.

For symmetrical indentation $\left(\eta=0\right.$ and $\left.\beta_{\mathrm{r}}=\beta_{1}=\beta\right)$

$$
L_{\text {contact }}=\frac{z_{\mathrm{d}}}{\cos \beta-\sqrt{2} \sin \varphi \sin \delta}
$$

$$
\begin{aligned}
& L_{\text {lip }}=\frac{z_{\mathrm{d}} \sqrt{2} \sin \varphi}{\cos \beta-\sqrt{2} \sin \varphi \sin \delta} \\
& 2 \sin ^{2} \varphi \sin \delta \cos \delta+2 \sqrt{2} \sin \varphi \sin \beta \sin \delta \\
& \quad-\sin \beta \cos \beta=0
\end{aligned}
$$

